\title{
EchoGéo
}

$9 \mid 2009$

L'Asie centrale : le temps des recompositions

\section{Le patrimoine urbain en Asie centrale}

\section{Guillemette Pincent}

\section{OpenEdition}

Journals

Édition électronique

URL : https://journals.openedition.org/echogeo/11220

DOI : 10.4000/echogeo. 11220

ISSN : 1963-1197

\section{Éditeur}

Pôle de recherche pour l'organisation et la diffusion de l'information géographique (CNRS UMR 8586)

\section{Référence électronique}

Guillemette Pincent, «Le patrimoine urbain en Asie centrale », EchoGéo [En ligne], 9 | 2009, mis en ligne le 17 juin 2009, consulté le 01 août 2021. URL : http://journals.openedition.org/echogeo/11220 ; DOl : https://doi.org/10.4000/echogeo.11220

Ce document a été généré automatiquement le 1 août 2021

EchoGéo est mis à disposition selon les termes de la licence Creative Commons Attribution - Pas d'Utilisation Commerciale - Pas de Modification 4.0 International (CC BY-NC-ND) 


\title{
Le patrimoine urbain en Asie centrale
}

\author{
Guillemette Pincent
}

\section{Introduction}

1 En latin, "patrimonium » signifie "l'héritage du père ». Aujourd'hui, le "patrimoine " est défini plus largement comme «le bien d'héritage qui descend, suivant la loi, des pères et des mères à leurs enfants ${ }^{1} »$ : le patrimoine, hérité du passé, se transmet. C'est le cas du "patrimoine urbain ", qui regroupeselon F. Choay " les tissus, prestigieux ou non, des villes et ensembles traditionnels pré-industriels et du XIX ${ }^{\mathrm{e}}$ siècle, et tend à englober de façon plus générale tous les tissus urbains fortement structurés» (Merlin, Choay, 2000, p. 580). Pour qu'il y ait transmission, le patrimoine doit être entretenu, protégé. À l'échelle de la ville, l'acteur politique joue alors un rôle primordial : c'est lui qui détermine quel héritage peut être préservé et accéder ainsi à l'historicité. Selon J. Monnet, «la protection du patrimoine est une opération ségrégative qui tend à privilégier, à soumettre à une règle spécifique des espaces déterminés en fonction des intérêts de l'Etat » (Monnet, 1993, p. 109) : en choisissant de préserver certaines constructions, le pouvoir politique leur reconnaît un droit à perdurer en tant qu'héritage. Il marque ainsi l'espace et lui donne une nouvelle valeur. Par-là même, il affirme son emprise territoriale. Le «patrimoine urbain" est donc considéré dans cet article comme un tissu urbain fortement structuré, hérité du passé puis transmis aux générations futures grâce à l'action de l'Etat. Cette expression occidentale utilisée à partir de la fin du XIX siècle (Choay, 1996, p. 132) puis proposée pour la première fois par G. Giovannoni en 1931, a-t-elle un sens en Asie centrale ? Cette question, un brin provocatrice, mérite cependant d'être posée : au-delà des nuances linguistiques, qu'est-ce que le patrimoine urbain centrasiatique? Quels sont les «tissus urbains fortement structurés » hérités des siècles passés et mis en valeur par l'Etat? Pour quelles raisons acquièrent-ils le droit à l'historicité ?

Notre définition de l'Asie centrale fait référence à une zone géographique et culturelle ${ }^{2}$ qui inclut à l'est la région autonome du Xinjiang, située en Chine, et à l'ouest, les cinq 
ex-républiques socialistes et soviétiques: Kazakhstan, Kirghizstan, Ouzbékistan, Tadjikistan, Turkménistan. À l'intérieur de cet ensemble régional, il nous faut distinguer le nord de l'Asie centrale de tradition davantage nomade, aux plaines et bassins du sud de l'Asie centrale, aux populations largement et précocement sédentarisées. Les enjeux liés à la préservation du patrimoine bâti sont ainsi beaucoup moins prégnants au Kazakhstan et Kirghizstan - des régions plus tardivement urbanisées et dans lesquelles les vestiges des cités anciennes ne sont plus que ruines ou ont disparu (Chymkent, Otrar au Kazakhstan) -, que dans les oasis plus méridionales, où les vestiges du passé sont encore visibles aujourd'hui, principalement en Ouzbékistan (par exemple: Boukhara, Khiva, Kokand, Samarcande, Tachkent) et dans le Xinjiang (par exemple : Kachgar, Kokand, Turfan, Yarkand). C'est pour cette raison que dans cet article, nous nous concentrons sur les espaces urbains de ces deux régions.

La ville y apparaît comme un palimpseste sur lequel se sont déposées les traces de chaque régime politique : jusqu'au XIX ${ }^{e}$ siècle, les khan $^{3}$ locaux règnent sur leurs terres. Mais en 1867, les Russes conquièrent Tachkent, prélude à la colonisation dans le Turkestan occidental ${ }^{4}$ tandis qu'en 1876 , le Turkestan oriental ${ }^{5}$ est sous domination mandchoue puis chinoise à partir de 1911. Vient l'époque marquée par l'idéologie communiste : de 1917 à 1991 à l'ouest du Pamir, et de 1949 jusqu'à nos jours en Chine. Depuis la chute de l'URSS en 1991, les ex-Républiques socialistes et soviétiques devenues indépendantes sont dirigées par des mains plutôt autoritaires (Laruelle, Peyrouse, 2006) qui cherchent à consolider les identités nationales ${ }^{6}$, tandis que la République autonome du Xinjiang créée en 1955 est régulièrement agitée de soubresauts indépendantistes animés par les populations ouïgoures ${ }^{7}$ : ces deux configurations politiques influencent-elles la place accordée par l'Etat au patrimoine urbain?

Cette analyse, qui se veut générale et synthétique, repose sur un travail de terrain réalisé en Asie centrale de 2003 à 2008, principalement en Ouzbékistan et dans le Xinjiang. Les informations recueillies (entretiens, enquêtes, observation non participante) ont été recoupées avec les sources bibliographiques tant théoriques ${ }^{8}$ qu'appliquées à la région étudiée. Les données statistiques, peu nombreuses et peu fiables, ne sont que peu utilisées. Un point méthodologique important doit enfin être souligné : l'objectif de cet article n'est pas de vouloir plaquer une notion occidentale à un territoire qui ne l'est pas, mais plutôt de proposer des éléments favorables à une compréhension mutuelle.

\section{Les héritages urbains centrasiatiques à l'épreuve de la sélection}

\section{La sélection chronologique : la mémoire au service de l'idéologie}

Selon F. Choay, nous assistons depuis les années 1960 à une inflation chronologique : en Europe occidentale, tout élément du passé, devient «patrimoine " (Choay, 1996, p. 9). M. Guillaume parle même d'" une nouvelle forme de passion du passé » qui « semble saisir les sociétés industrielles de l'Occident» (Guillaume, 1980, p.11). La cité d'antan est automatiquement considérée comme "patrimoine urbain». Mais en Asie centrale, l'éventail chronologique est beaucoup plus restreint: ce qui vient du passé n'est pas forcément considéré comme un «patrimoine » à protéger et à transmettre. Parmi les 
«tissus urbains fortement structurés » qui ont jusqu'à présent traversé les ans, voire les siècles, seuls quelques-uns ont droit à l'historicité.

6 La ville centrasiatique est un palimpseste sur lequel nous pouvons lire différentes structures urbaines, qui chacune forme aujourd'hui un héritage encore visible. La plus ancienne, appelée Eski Chahar («vieille ville » en ouzbek) ou Lao Cheng ("vieille ville » en chinois) est formée de rues étroites, labyrinthiques, qui se terminent souvent en impasse. Les maisons en brique crue, organisées autour de leur cour, n'offrent à la vue des passants que des façades aveugles. Le tissu urbain, ponctué de monuments religieux ou commerciaux, autrefois entouré de remparts percés de portes, est structuré autour du pôle de la mosquée. L'eau est omniprésente dans ces oasis: elle coule dans les canaux d'alimentation et d'évacuation (les aryk ouzbeks), circule dans des galeries souterraines (les qanat ou karez ouïgours), se repose dans des bassins ou dans de profonds puits. En Ouzbékistan, à Boukhara, à l'intérieur des vestiges des derniers remparts, les quartiers résidentiels s'articulent autour de l'ensemble religieux Kalon et de l'ancien bazar qui se déployait entre trois coupoles marchandes (Tok-i zargaran, Tok-i tilpak-foruchan, Tok-i sarafan), non loin du canal central et des nombreux khauz ${ }^{9}$.

7 À partir de la fin du XIX siècle, une nouvelle strate urbaine se juxtapose à la précédente. C'est le cas dans le Turkestan occidental alors sous domination russe. À Tachkent, Yangi Chahar ("ville nouvelle » en ouzbek), se développe à l'est d'Eski Chahar. Les rues sont plus larges, géométriques, les bâtiments plus massifs et plus hauts, des arbres sont plantés. L'architecture russe y est encore visible, que ce soit à travers quelques monuments (Résidence Romanov à Tachkent) ou dans certains quartiers résidentiels où s'alignent les maisons en $\mathrm{R} / \mathrm{R}+1$, aux toits à double pente et aux fenêtres ouvertes sur la rue. Le même type de structure urbaine se retrouve aujourd'hui dans de grandes villes telles que Douchanbé (environ 679400 habitants en 2008), la capitale du Tadjikistan, ou de plus petites comme Karakol (64 322 habitants au recensement de 1999), située à l'est du lac Issik Koul au Kirghizstan.

8 Tout au long du XXe siècle, les principes socialistes prônés à la fois par l'URSS au sein de ses Républiques socialistes puis par la République populaire de Chine, s'expriment dans l'espace urbain centrasiatique : les avenues parcourues de transports en commun et les immeubles d'habitations collectives de plus en plus hauts (de $R+3$ à $R+16)$ répondent au souhait d'une société unifiée et égalitaire. De plus, la ville est considérée comme un espace de production: les industries sont implantées au cœur des cités (Korugh au Tadjikistan) ou des villes nouvelles sont créées autour de pôles économiques (Karaganda au Kazakhstan, en 1934). L'idéologie s'impose : les bâtiments administratifs sont monumentaux, de même que les places sur lesquelles ont régulièrement lieu des rassemblements autour de la statue de Mao (Kachgar) ou de Lénine (place Lénine à Tachkent).

9 Depuis la chute de l'URSS et la création de Républiques indépendantes à l'ouest du Pamir, depuis l'ouverture de la Chine à l'économie de marché dans les années 1990, la ville centrasiatique se pare des attributs de nouvelles idéologies : dans le Xinjiang, le paysage urbain d'Urumqi, la capitale de la région autonome, est rythmé par la construction de gratte-ciels. Au Kazakhstan, Almaty est parcourue de voitures rutilantes qui déambulent dans des rues vouées au capitalisme émergent. Certaines capitales des anciennes républiques socialistes et soviétiques sont devenues les vitrines d'un pays désormais indépendant. Elles sont utilisées pour renforcer l'identité nationale et servir l'idéologie locale. Achgabat en est la caricature : la capitale du 
Turkménistan a été façonnée pour répondre aux souhaits les plus fantasques de son exPrésident, Turkmenbachi, qui n'a pas hésité à construire une cité à son effigie (Gintrac, Fenot, 2005).

10 Parmi ces tissus urbains, seuls quelques-uns sont mis en valeur par l'Etat. Ils sont alors considérés comme des "patrimoines » dignes d'être transmis, aptes à l'historicité. En Ouzbékistan, les bâtiments de la période précoloniale sont bien plus protégés que ceux issus de la période coloniale et soviétique. La liste des monuments historiques du pays montre que les édifices protégés par l'Etat datent principalement des XV, XVI et $\mathrm{XIX}^{\mathrm{es}}$ siècles. Certaines périodes de l'histoire sont donc davantage mises en valeur que d'autres et la période timouride ${ }^{10}$ (XIV-XVI ${ }^{\mathrm{e}}$ siècle) domine : à Boukhara, la medresseh ${ }^{11}$ Ulug Beg (1417), inscrite sur la liste des monuments historiques, a subi des travaux de ravalement en 1994 à l'occasion du 600 anniversaire d'Ulug Beg, petit-fils de Tamerlan ${ }^{12}$. D'autres bâtiments situés à proximité n'ont toujours pas été restaurés.

11 La plupart des emblèmes de la période coloniale et de l'URSS ont disparu. Les monuments construits pendant la période tsariste sont rares (Palais Romanov à Tachkent) et les quartiers d'habitations ont été profondément remaniés (tout autour de la place Tamerlan, par exemple). De nombreux cinémas construits entre 1924 et 1991, symboles architecturaux de cette époque, ont été détruits. C'est le cas à Samarcande, dans l'artère piétonne située entre le Registan et l'ensemble de Bibi Khanum ${ }^{13}$. Sauf quelques rares exceptions (principalement au Kirghizstan), les statues de Lénine ont disparu et les monuments dédiés aux héros soviétiques sont peu à peu détruits ou déplacés. Il y a quelques semaines, le monument qui louait l'amitié entre les peuples, situé en plein cœur de Tachkent, a été installé dans un faubourg lointain ${ }^{14}$.

Concernant la période post-soviétique, nous manquons de recul pour apprécier ce qui est considéré comme "patrimoine». Nous pouvons cependant souligner que les bâtiments construits à Achgabat en l'honneur de feu Turkmenbachi sont en grande partie conservés et vénérés malgré la mort du guide de la nation en 2006. En Ouzbékistan, certains lieux emblématiques de l'indépendance de la République sont eux aussi inscrits sur la liste des monuments historiques. C'est le cas du musée Tamerlan et de la place Mustakillik ${ }^{15}$ à Tachkent. Les symboles architecturaux des idéologies postsoviétiques et certains héritages précoloniaux sont donc pris en compte par l'Etat en tant que patrimoine urbain à préserver : le reste ne compte pas.

\section{Le choix typologique : l'éloge du monument}

Selon F. Choay, le patrimoine bâti fait l'objet en Europe occidentale d'une inflation chronologique - on l'a vu - mais aussi typologique (Choay, 1996). Un monument historique tout comme une fontaine, un lavoir, une maison, peuvent être considérés comme "patrimoine»: le prestige et le vernaculaire ont tout autant le droit à l'historicité. En Asie centrale, l'éventail typologique est nettement moins large et s'applique principalement au monument. Défini par F. Choay comme «l'artefact qui nous interpelle pour nous faire ressouvenir ${ }^{16}$ ", il regroupe ici quatre types différenciés par leur usage principal : les monuments religieux (mosquée, medresseh, mausolée), commerciaux (coupole marchande), politiques ( $\left.a \mathrm{rk}^{17}\right)$ et hydrauliques (bassins, canaux). En Asie centrale, le monument fait l'objet d'un soin particulier de la part des autorités nationales : à l'est du Pamir, dans le Xinjiang, il est la cible sur laquelle se concentre l'action de l'Etat chinois pour assurer son emprise territoriale et culturelle. Une plaque 
dorée orne la grande mosquée de Kachgar (mosquée Idkah, $\mathrm{XV}^{\mathrm{e}}$ siècle), seul monument réhabilité de la ville. Elle explique que l'Etat chinois a réhabilité le bâtiment en 2001. L'esplanade autrefois pleine de commerces et d'animation est désormais surmontée d'un écran géant sur lequel sont diffusées les chaines de télévision nationale : le chant des prières a fait place à la musique des groupes de rock chinois. A l'inverse, les héritages vernaculaires, plus discrets, ne sont que très rarement envisagés comme des éléments de patrimoine alors qu'ils constituent l'essentiel du tissu urbain centrasiatique. À Kachgar, dans le Xinjiang, les faubourgs de Lao Cheng ont été en partie détruits.

Deux nuances doivent toutefois être apportées : tous les monuments centrasiatiques ne bénéficient pas du même traitement de faveur. Seuls ceux utiles et sélectionnés en tant que tel par l'Etat ont droit à l'historicité. Enfin, faute de moyens financiers, la plupart de ces édifices, même officiellement protégés par les autorités nationales, ne sont pas entretenus : il est impossible pour les pouvoirs publics de tous les réhabiliter. L'Etat doit donc procéder à des choix, et il le fait conformément à ses aspirations.

Définir ce qu'est le patrimoine en Asie centrale est un exercice périlleux : l'approche chronologique nous montre que tout héritage bâti n'est pas forcément considéré comme un élément digne d'intérêt, l'approche typologique souligne le caractère à la fois restreint et fluctuant de la notion de patrimoine urbain, qui s'applique aux monuments plutôt qu'aux constructions vernaculaires, à ceux qui ont une portée politique actuelle plutôt qu'à ceux hérités d'une période plus sombre.

\section{Les valeurs du patrimoine urbain en Asie centrale}

\section{Les héritages urbains : des outils politiques}

Les héritages urbains deviennent des éléments de patrimoine lorsqu'ils acquièrent une utilité politique. S'ils vont à l'encontre de l'idéologie dominante, ils sont détruits. S'ils portent les idéaux nationaux, ils sont valorisés: selon J-Y. Andrieux, «le patrimoine, notion vivante et évolutive, longuement auscultée ici, peut continuer de jouer un rôle éminent dans l'évolution des sociétés et des pays aussi bien qu'il peut récupérer les dérives ou les surenchères nationalistes, des plus graves aux plus dérisoires " (Andrieux, 1997, p. 214). Ces mécanismes sont très visibles en Asie centrale et ne sont pas nouveaux. Depuis la fin du $\mathrm{XIX}^{\mathrm{e}}$ siècle, les pouvoirs politiques qui se sont succédé ont participé, chacun à leur façon, à la patrimonialisation centrasiatique. Le cas est particulièrement visible en Ouzbékistan (Pincent, 2008, I) : lors de la période tsariste, les héritages précoloniaux, pour la plupart en ruines, ne sont guère pris en compte par les autorités locales. À Tachkent, les remparts et les portes d'Eski Chahar sont détruits mais la vieille ville continue de fonctionner, irriguée par des rues tortueuses et articulée autour de son bazar central, Chorsou. Les Russes considèrent le territoire centrasiatique comme un espace économique à contrôler et pratiquent la juxtaposition spatiale : de l'autre côté du canal, ils construisent Yangi Chahar, la ville nouvelle, dans laquelle sont implantées des installations militaires, prémisses de la future cité. La prise en compte des héritages précoloniaux est beaucoup plus forte et plus ambiguë pendant la période soviétique. Alors qu'est mise en place l'esquisse d'un cadre juridique de protection patrimoniale, les monuments religieux présents dans les ex-républiques socialistes sont anéantis ou leurs usages sont transformés afin de mieux correspondre aux préceptes communistes : 
les caravansérails deviennent des habitations collectives, les mosquées des cinémas. Seules quelques medresseh ou mosquées sont réhabilités, mais leur survie ne vaut que si elles sont mises au service de l'idéologie dominante. À Samarcande, l'ensemble du Registan est déblayé, les minarets sont redressés, mais ces monuments perdent leur usage religieux : sur la place centrale sont brûlés les voiles des femmes. Le monument est alors considéré comme un emblème politique, symbole d'une nouvelle société.

À partir des années 1950, la perception des héritages précoloniaux par les autorités locales et fédérales évolue: les édifices qui ont traversé les siècles ont désormais un rôle économique. Ils permettent d'une part de remédier à la crise du logement qui sévit et d'autre part, d'être les supports d'un développement touristique fédéral puis international; quelques monuments préalablement sélectionnés sont la preuve officielle et éclatante que les autorités politiques respectent les grands principes de protection patrimoniale énoncés dans les chartes et conventions internationales (1964: Charte de Venise, 1972: Convention de l'UNESCO). Et puis l'URSS disparait et de nouvelles républiques naissent, impatientes de retrouver coûte que coûte les traces de leur glorieux passé. Le monument, support d'une mémoire collective partagée (Halbwachs, 1997), est alors considéré dans ces pays indépendants comme un marqueur puissant et visible de l'espace urbain, comme le symbole d'un passé commun dénué de toute domination exogène : il constitue une justification historique au nouvel Etat. En Ouzbékistan, Tamerlan est considéré comme un héros national, figure forte du pouvoir qui renvoie à celle d'I. Karimov: nous comprenons aisément que les monuments timourides soient réhabilités en priorité tandis que ceux hérités de la période russe ou soviétique soient occultés, voire détruits, symboles d'un passé encore trop proche, dominé par des puissances exogènes.

\section{Eski Chahar et Lao Cheng: des leviers économiques}

Eski Chahar et Lao Cheng acquièrent un droit à l'historicité lorsque l'Etat leur reconnaît une valeur économique. À Kachgar, deux quartiers de Lao Cheng subsistent encore au cœur de la ville. Ils sont protégés et l'entrée y est payante. Ils sont encore habités mais voués à un usage principalement touristique, étroitement contrôlé. Les maisons servent de boutiques d'artisanat ou de restaurant (Figure 1). Le tissu urbain est donc préservé à une condition : qu'il rapporte. 
Figure 1 - La valorisation touristique d'une habitation à Kachgar

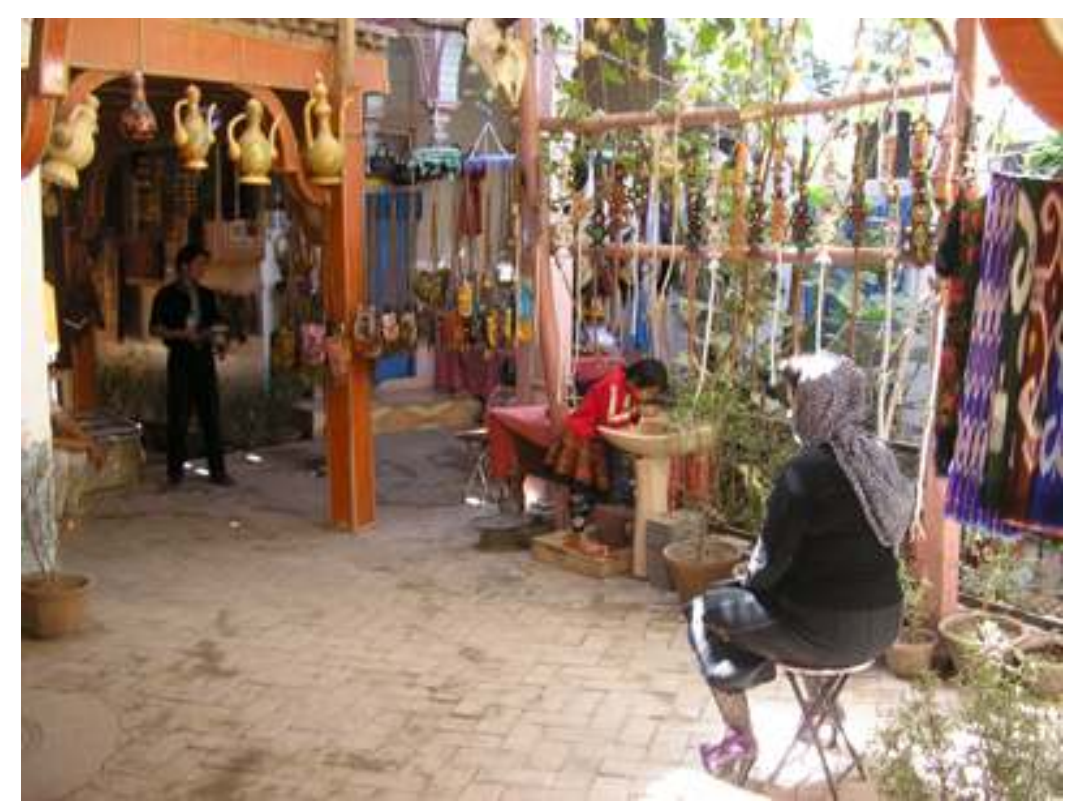

Source : G. Pincent, 2006

Dans la cour d'une habitation récemment réhabilitée, une famille vend quelques articles d'artisanat de mauvaise facture. La visite de cette cour est une des étapes obligées pour les touristes, encadrés par des guides locaux très attentifs aux moindres déplacements de leurs hôtes dans le quartier ouïgour.

Dans des pays où le PNB par habitant ne dépasse pas toujours 1300 US\$ ${ }^{18}$, la protection des héritages historiques n'est pas prioritaire. Au contraire. Souvent très coûteuse, elle n'est envisagée qu'à une condition: le patrimoine doit rapporter. La valorisation touristique est alors primordiale. En Ouzbékistan, à Khiva, la vieille ville Ichan Kala est un musée à ciel ouvert destiné aux voyageurs, qui payent pour entrer dans la cité entourée de hauts murs. Ce procédé n'est pas nouveau: Ichan Kala a été en partie reconstruite à des fins scientifiques et touristiques dès la fin des années 1960. Les projets de réhabilitation réalisés depuis 2006 à Boukhara sont dus à la volonté politique du ministère de la culture, de la compagnie nationale du tourisme et de la municipalité boukhariote de faire de cette ville la capitale culturelle de l'Ouzbékistan ${ }^{19}$ : il faut dire qu'en 2004 l'activité touristique représente à l'échelle nationale près de 57 millions de US\$ de revenus ${ }^{20}$. Le bâtiment coûte ou rapporte, et c'est aussi selon ces critères que les acteurs décident ou pas de transformer un édifice. X. Greffe traite des aspects économiques de l'héritage bâti : "L'existence du patrimoine monumental génère en effet de nombreux flux économiques à l'occasion de sa constitution, de son exploitation ou de sa rénovation" (Greffe, 1990, p. 2). Les retombées économiques des travaux, la transformation des usages et du cadre bâti, l'image de marque renouvelée contribuent à donner un nouvel élan aux héritages urbains : le tourisme joue un rôle majeur dans la mise en valeur d'Eski Chahar et de Lao Cheng, au point d'être parfois perçu comme une véritable manne financière qui permet par exemple de moderniser les infrastructures routières et d'alimentation en eau... Souvent, il est vrai, au prix de destructions de certaines habitations. À Tachkent, des rues ont été élargies à partir de 1992 afin de réguler la circulation aux alentours du bazar Chorsou et d'installer des canalisations jusqu'alors inexistantes ou défectueuses. À cette occasion, les maisons qui se trouvaient 
en bordure de route ont été détruites. Mais celles qui ont été conservées ont désormais l'eau courante.

\section{Une valeur historique mise au ban?}

20 Nous comprenons aisément que la valeur historique des héritages urbains centrasiatiques n'a souvent que peu de poids face aux impératifs économiques et politiques de l'Etat : ce qui est ancien, vieux, daté, n'acquiert pas systématiquement le droit à la protection, à la réhabilitation, à la postérité. On l'a vu, des pans entiers d'Eski Chahar et de Lao Cheng sont détruits, des monuments - même officiellement protégés sont abandonnés. Par ailleurs, l'authenticité historique n'a pas la même importance qu'en Europe occidentale. Peu importe qu'un monument soit détruit puis reconstruit sans respect de son architecture initiale : ce qui compte est le respect de la mémoire. Des enquêtes menées à Boukhara entre 2004 et 2005 montrent les réactions contrastées des habitants face aux réhabilitations menées par l'Etat dans Eski Chahar. Cela ne choque pas que les usages d'un monument évoluent. Mais lorsque ces transformations ne sont pas conformes à la fonction initiale, là, les contestations sont vives: une medresseh peut être utilisée comme lieu d'enseignement, même non religieux. Mais qu'une mosquée soit transformée en hôtel, c'est "cracher dans sa mémoire ${ }^{21}$ ». Les héritages bâtis ont une dimension immatérielle très forte: des mausolées, même en ruines, sont encore utilisés pour le culte ${ }^{22}$. La mémoire des lieux est plus importante que la valeur historique des briques. Ces dernières peuvent disparaître, pas la mémoire.

\section{Les différents types de mises en valeur du patrimoine urbain centrasiatique}

21 Les modes de préservation des héritages urbains centrasiatiques obéissent à des approches très variées qui ont un point commun : la sélection.

\section{La transformation des usages}

22 À Khiva, en Ouzbékistan, la medresseh M. A. Khan est un hôtel-restaurant. À Boukhara, la medresseh Koukeldach est remplie de commerces. À Turfan, dans le Xinjiang, la mosquée Imin est principalement visitée par les touristes, et non plus les pèlerins. Ce procédé, utilisé tout au long du XX $\mathrm{X}^{\mathrm{e}}$ siècle par les Soviétiques et les Chinois, a permis de conserver certains monuments en les adaptant à des usages plus conformes à l'idéologie d'alors (Pincent, 2006): les fonctions religieuses sont effacées au profit d'activités socialistes. Selon P. R. Gaubatz, ce mécanisme ne s'applique pas qu'aux monuments. Il explique ainsi que dans le Xinjiang, "vernacular architecture has posed both spatial and ideological barriers to preservation. Since large private houses were socially unacceptable after the 1949 revolution, many were converted into multiple-family dwellings " (Gaubatz, 1996, p. 275). Aujourd'hui, ce processus perdure, mais il est mis au service du tourisme. En Ouzbékistan, le décret promulgué le 27 juillet 1992 encourage l'implantation d'échoppes artisanales dans les monuments historiques: ces activités permettent de dégager des sources de revenus destinés officiellement à l'entretien des édifices, tout en proposant des souvenirs aux touristes. 


\section{La reconstruction}

La reconstruction est un procédé très utilisé en Asie centrale ${ }^{23}$. Elle repose parfois sur des éléments historiques avérés, déterminés par des fouilles archéologiques précises (reconstruction du Tok-i sarafan en 199724), mais elle est très souvent réalisée sans souci de l'authenticité historique. Les exemples sont nombreux : à Tachkent, l'ensemble Khazrati Imam (XVI ${ }^{e}$ siècle) a été presque entièrement reconstruit en 2006 (Pincent, 2008) (Figure 2). À Turfan, les touristes sont invités à visiter des galeries souterrainesreconstituées en sous-sol. La promenade est rythmée par les boutiques de souvenirs. À Samarcande, l'ensemble religieux du Châh-i-Zinde (XIV ${ }^{e}$ siècle) a été profondément remanié en 2006 : des tombeaux ont été reconstruits et presque tous les bâtiments ont été restaurés selon des procédés techniques fort décriés. La nécropole parée de « carrelage » multicolore est désormais surnommée la « salle de bain ».

Figure 2 - La (re)construction de la nouvelle Grande mosquée de Tachkent, à droite

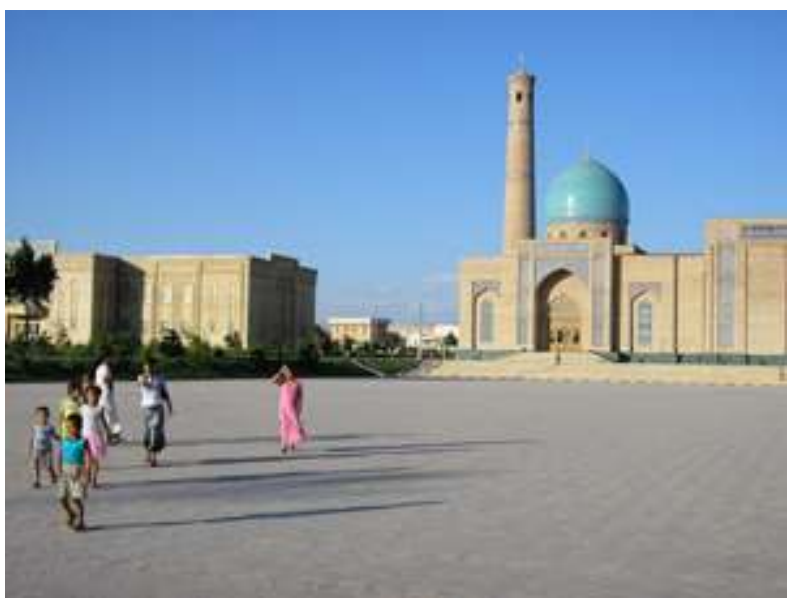

Source : G. Pincent, 2007.

$\mathrm{Au}$ fond à droite, l'architecture de la mosquée construite en 2006 est fortement inspirée de la période timouride. Au centre, une immense esplanade traversée par les habitants des quartiers alentours. A cet emplacement se trouvaient une école et une bibliothèque édifiée au XIX ${ }^{\mathrm{e}}$ siècle qui abritait le Coran d'Osman, considéré comme le plus ancien du monde.

\section{La destruction sélective}

Pour mettre en valeur certaines parties d'Eski Chahar ou de Lao Cheng, les autorités locales ou nationales n'hésitent pas à en détruire des pans entiers. C'est ce qui s'est passé à Kachgar, autour de la Cité des Potiers : les faubourgs sont détruits pour que de nouvelles infrastructures de transport soient créées. Les héritages vernaculaires ne sont alors considérés que comme une gêne à la modernisation du tissu urbain ou à la réhabilitation d'un monument plus grandiose. À Samarcande, la restauration du Gur Emir a entraîné la destruction d'une partie du quartier qui l'entourait. La première tranche des travaux, qui s'est étalée de 1991 à 2000, a permis de dégager de larges perspectives vers le tombeau resplendissant de Tamerlan tandis que subsistent quelques maisons dissimulées derrière un mur. Cette démarche, utilisée il y a peu en Europe occidentale, provoque encore de vifs débats: J-P. Babelon et A. Chastel 
affirment qu' "un monument n'est jamais isolé; la pire erreur du siècle dernier fut sans doute d'ignorer la continuité du tissu urbain, de multiplier les grands vides, de régulariser les grandes places à tout prix" (Babelon, Chastel, 1994, p. 99). La destruction est pourtant une méthode couramment utilisée pour valoriser Eski Chahar ou Lao Cheng car elle permet de créer des perspectives architecturales ou techniques.

\section{La folklorisation et l'invention culturelle}

Une dernière approche consiste à «inventer " une culture au nom d'impératifs économiques et politiques. En Ouzbékistan, à Boukhara, certains souhaitent reconstruire Eski Chahar pour que cette dernière ressemble à un décor des " 1001 nuits ", sans rapport avec ce qui existait autrefois ${ }^{25}$. Selon eux, la cité serait alors plus attractive pour les touristes. Pour créer ce nouveau paysage urbain, des architectes prônent le style néo-ouzbékistanais, fusion entre des matériaux modernes et des formes d'inspiration arabo-musulmane (Figure 3). Dans un contexte de renforcement identitaire lié à l'indépendance de la jeune République (1991) et à un pouvoir actuellement fort, la priorité est de créer une culture unificatrice, qui plus est, capable d'attirer les touristes.

Figure 3 - Un exemple du style néo-ouzbékistanais dans un hôtel de Boukhara

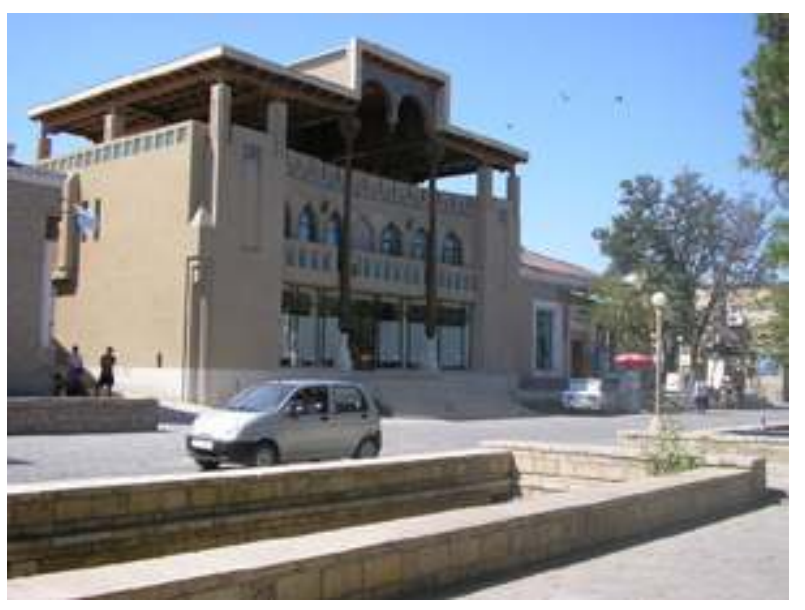

Source : G. Pincent, 2007.

La façade de cet hôtel situé au cœur d'Eski Chahar, tout près du bassin du Liabi-khauz (XVII' siècle), s'inspire de l'architecture des ä̈van ${ }^{26}$ traditionnels tout en intégrant des matériaux récents.

Dans le Xinjiang, la logique est quelque peu différente: face aux revendications indépendantistes des Ouïgours, le pouvoir central chinois cherche à canaliser la culture de cette minorité. Deux mécanismes sont utilisés: la folklorisation simplifie en quelques traits la complexité de la culture ouïgoure, puis l'appauvrit, tandis que la recherche du syncrétisme sino-ouïgour, en inventant un nouveau style architectural, brouille les identités. Les méthodes diffèrent mais le principe est le même: marquer l'espace urbain de son règne, transformer la ville selon son idéologie. L'espace, en tant que support de la mémoire collective (Halbwachs, 1997), est un outil politique à contrôler. À Turfan, les karez reconstruits en béton sont ponctués de boutiques de souvenirs. Des spectacles de danses folkloriques, très prisés des groupes de touristes Hans, sont organisés au milieu des vignes transformées en lieux de déambulation et de 
dégustation. À Yarkand, la place située devant la Grande mosquée est aujourd'hui entourée de bâtiments à l'architecture d'inspiration arabo-musulmane tandis que des ponts typiques des jardins chinois se dressent sur l'esplanade. La recherche du folklore et de ce syncrétisme n'est-elle pas le signe d'une culture ouïgoure caricaturée, reniée, dominée? La plupart des infrastructures touristiques construites pour valoriser certains quartiers des cités ouïgoures - ceux qui n'ont pas encore été détruits - sont détenues par des Han. De la même manière, les karez de Turfan ou la mosquée Idkah sont majoritairement visités par des non-Ouïgours. Seule l'activité touristique permet et justifie aux yeux des autorités nationales la préservation des quelques lambeaux de Lao Cheng.

\section{Le rôle limité des acteurs internationaux}

Dans ce contexte, la question des acteurs de la préservation des héritages bâtis prend un sens particulier: en Asie centrale, dans des pays dirigés depuis dès des décennies par des pouvoirs forts et parfois dictatoriaux, la question patrimoniale est avant tout une affaire d'Etat. Que ce soit dans le Xinjiang ou en Ouzbékistan, seules les plus hautes personnalités décident des travaux majeurs de réhabilitation ou de destruction d'Eski Chahar ou de Lao Cheng. Pourtant, elles doivent parfois jouer avec les organisations internationales telles que l'UNESCO. Le font-elles vraiment? En Ouzbékistan, la plupart des programmes de coopérations qui visent à préserver des héritages bâtis se terminent en divorce. La Fondation Agha Khan n'y travaille plus: sa manière de travailler n'a pas plu à l'Etat ouzbékistanais. Quant à l'UNESCO, son rôle est bien souvent très limité : sa marge de manœuvre est nulle dans les quartiers qui ne sont pas inscrits sur la liste du patrimoine mondial, et très limitée dans les sites inscrits. Elle apporte un soutien financier non négligeable mais n'a pas toujours le temps ni les moyens d'intervenir ou de remettre en cause les projets impulsifs du gouvernement. Lors de la destruction des quartiers situés autour du Gur Emir, à Samarcande, l'UNESCO, bien que prévenue par des habitants, n'a pu réagir: les travaux ont été réalisés en quelques jours. Elle n'a rien pu faire non plus lors de la rénovation fort controversée de la nécropole Châh-i-Zinde. Réduite à l'inaction au nom de la diplomatie, elle n'a pu que proférer la menaced'inscrire la ville de Samarcande sur la liste du patrimoine en péril sans pour autant la mettre à exécution.

\section{Conclusion}

La prise en compte des héritages urbains par les autorités nationales centrasiatiques perturbent notre vision euro-centrée et souligne toute la relativité de la notion de patrimoine, fluctuante selon les époques et les territoires. En Asie centrale, le patrimoine urbain est le résultat d'un choix étatique avant tout lié à des impératifs politiques et économiques. Il s'agit d'un héritage bâti qui rapporte de l'argent, qui est conforme à une idéologie et acquiert ainsi une fonction bien plus symbolique qu'historique. Cette approche centrasiatique peut gêner nos perceptions: des organisations internationales telles que l'UNESCO tentent vainement de faire entendre leur voix auprès des gouvernements pour que la notion d'authenticité soit respectée, pour que la valeur historique de certains héritages bâtis soient préservée. Mais même à Samarcande, partiellement inscrite depuis 2001 sur la liste du patrimoine mondial de 
l'UNESCO, des pans entiers d'Eski Chahar sont détruits, des monuments sont restaurés ou reconstruits sans grand souci d'authenticité. Cette analyse synthétique souligne la difficulté - et le risque - d'appliquer une notion exogène à un territoire qui a son propre fonctionnement, ses propres règles. Elle nous montre que tout n'est pas patrimoine dans le passé : il est possible de valoriser les cités historiques sans vouloir atteindre des objectifs de protection patrimoniale, il est fréquent que soient sauvegardés des monuments qui, maintes fois détruits puis reconstruits, ont perdu toute authenticité.

\section{BIBLIOGRAPHIE}

Andrieux J. Y., 1997, Patrimoine et histoire, Paris, Belin, 283 p.

Babelon J-P., Chastel A., 1994, La notion de patrimoine, Paris, Liana Levi, 142 p.

Choay F., 1996 (première édition : 1992), L'allégorie du patrimoine, Paris, Seuil, 244 p.

Gaubatz P. R., 1996, Beyond the Great Wall : urban form and transformation on the Chinese frontiers, Stanford (EU), Stanford University Press.

Gintrac C., Fenot A., 2005, Achgabat, une ville ostentatoire : autocratie et urbanisme du Turkménistan? Paris, L'Harmattan, $128 \mathrm{p}$.

Giroux A., avril 1994, Les Etats d'Asie centrale face à l'indépendance : Ouzbékistan, république kirghize, Tadjikistan, Turkménistan, Courrier des pays de l'Est, n 388, p. 3-43.

Greffe X., 1990, La valeur économique du patrimoine : la demande et l'offre des monuments, Paris, Anthropos, 253 p.

Guillaume M., 1980, La politique du patrimoine, Paris, Galilée, 196 p.

Halbwachs M., 1997 (première édition : 1950), La mémoire collective, Paris, Albin Michel, 295 p.

Komatsu H., 1991, Central Asia. Islamic urban studies, Tokyo, Masashi Manada and Toru Miura, p. 281-345.

Laruelle M., Peyrouse S., 2006, Asie centrale, la dérive autoritaire. Cinq républiques entre héritage soviétique, dictature et islam, Paris, Autrement, $135 \mathrm{p}$.

Lipovsky I., 1996, Central Asia in search of a new political identity, Middle East Journal, vol. 50, $\mathrm{n}^{\circ} 2$, p. 211-223.

Loubes J-P., 1998, Architecture et urbanisme de Turfan. Une oasis du Turkestan chinois, Paris, L'Harmattan, $433 \mathrm{p}$.

Merlin P., Choay F. et al., 2000, Dictionnaire de l'urbanisme et de l'aménagement, Paris, PUF, 903 p. Monnet J., 1993, La ville et son double. La parabole de Mexico, Paris, Nathan, 221 p.

Nekrasova E., Fillimonov V., 1998, L'ensemble architectural de Taq-e Sarrafjan à Boukhara, Cahiers d'Asie centrale, $n^{\circ}$ 5-6, p. 95-124. 
Nil'sen V. A., 1988, U istokov sovremennogo gradostroitel'stva Uzbekistana, XIX-načalo XX veka 瀶A l'origine de la construction moderne en Ouzbékistan, du XIX ${ }^{\mathrm{e}}$ au début du XX $\mathrm{XX}^{\mathrm{e}}$ siècle医, Tachkent, Gafur Guljam, 207 p.

Notkine M., 1981, Problèmes de protection et de reconstruction des monuments de l'architecture ancienne dans les villes historiques de l'Ouzbékistan, ICOMOS, www.international.icomos.org/monumentum/ vol14_2.pdf, 9 p.

Pincent G., 2008, La reconstruction des eski chahar à Tachkent et à Boukhara (Ouzbékistan) : à la recherche d'un mythe ?, Géographie et cultures, $n^{\circ} 65$, p. 15-32.

Pincent G., 2006, La réhabilitation fonctionnelle des villes précoloniales d'Ouzbékistan : un outil de domination politique ?, Cybergéo, http://www.cybergeo.presse.fr/eurogeo2.htm, 13 p.

Poujol C., 2001, Dictionnaire de l'Asie centrale, Paris, Ellipses, 352 p.

Roy O., 2001, L'Asie centrale contemporaine, Paris, PUF, coll. Que sais-je ?, 127 p.

Roy O., 1997, La nouvelle Asie centrale, ou la fabrication des nations, Paris, Seuil, 326 p.

Resul Y., 2002, The rebirth of Uzbekistan : politics, economy and society in the post-soviet era, Londres, Ithaca Press, 349 p.

Riegl A., 1984, Le culte moderne des monuments. Son essence et sa genèse, Paris, Seuil, 121 p.

Saliev A., 1991, Problemy rasselenija i urbanizacii v respublikah Srednej Azii 眮Les problèmes démographiques et d'urbanisation dans les républiques d'Asie centrale䜿, Tachkent, Fan, $112 \mathrm{p}$.

Suhareva O. A., 1962, Pozdnefeodal'nyj gorod Buhara konca XIX-načalo XX vv. 嘅La ville médiévale de Boukhara à la fin du XIX siècle-début du XXe siècle医, Tachkent, ANUzSSR, 193 p.

UNESCO, 1982, Conférence mondiale sur les politiques culturelles (26 juillet-6 août 1982). États et tendances des politiques culturelles des Etats membres d'Asie et du Pacifique, Paris, UNESCO, 17 p.

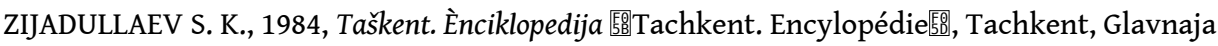
redakcija uzbekskoj sovetskoj ènciklopedii, $416 \mathrm{p}$.

\section{NOTES}

1. Définition du dictionnaire Littré, 1863.

2. Pour les définitions de l'Asie centrale, cf : Poujol, 2001, p. 39.

Komatsu, 1991, p. 281-345.

3. Khan : en ancien turc, "à l'origine, titre désignant le chef d'une confédération tribale, dérivé de la forme contractée kagan attestée sous la forme kan dans les inscriptions de l'Orkhon. Dans les siècles qui précèdent la conquête mongole, khân est un titre princier par opposition au titre suprême de sultan. Il finit par désigner le chef suprême de la formation étatique des Oügours, des Karakhanides et des Mongols. Dans les divers états issus de l'empire mongole, khân est le titre du souverain suprême. C'est aussi une particule honorifique ajoutée aux noms propres, ainsi que le terme local désignant le caravansérail.» In : Poujol, 2001, p. 159.

4. Turkestan (occidental) : "Division administrative fondée par la Russie en 1867 dont Tachkent est le siège et le général Von Kaufmann (1867-1881) le premier Gouverneur général.» In : Poujol, 2001, p. 311.

5. L'expression «Turkestan oriental» est utilisée par les partisans de l'indépendance de la région.

6. Sur la problématique de l'indépendance et de la construction identitaire des pays centrasiatiques :

Resul, 2002, p. 86-89 ; Lipovsky, 1996, p. 211-223 ; Giroux, 1994, p. 3-43. 
7. Ouïgour : «Peuple turcophone musulman ayant sa souche majoritaire en Chine (Xinjiang, plus de 7 millions) et une diaspora constituée de plusieurs strates au Kazakhstan et en Ouzbékistan (200000 personnes).» In : Poujol, 2001, p. 232 .

8. Par exemple : Riegl, 1984.

9. Khauz: en ouzbek, "un bassin, citerne, élément d'urbanisme très important dans les cités caravanières d'Asie centrale, généralement situé aux abords d'une mosquée pour permettre les ablutions rituelles, il est aussi destiné à la consommation urbaine. » In : Poujol, 2001, p. 161.

10. Timouride (période) : Tamerlan règne de 1369 à 1405. Puis de prestigieux souverains héritiers du conquérant lui succèdent jusqu'à l'arrivée à partir du XVIe siècle des clans ouzbeks chaybanides.

11. Medresseh : école coranique. L'écriture de ce terme varie : «madrasa» en langue arabe, «medresseh» ou «medreseh» en langues turciques.

12. Tamerlan : (1336-1405), régnant sur la Transoxiane à partir de 1369, il conquiert le Khorassan, l'Irak, l'Azerbaïdjan, puis Delhi, Alep, Bagdad et fait de Samarcande sa capitale, où il est enterré dans le tombeau du Gur Emir.

13. Entretien réalisé en français avec Mme C. Poujol, professeur à l'INALCO, le 25 septembre 2003, à Paris.

14. http://enews.ferghana.ru/article.php?id=2524, le 21/04/2009

15. Mustakillik : en ouzbek, « indépendance ».

16. Préface de Mme F. Choay, in : Riegl, 1984, p. 11.

17. Ark : en langues turciques, « citadelle ». La plus connue est la "citadelle de Boukhara, attestée depuis près de 2000 ans sur une colline artificielle de plus de quatre hectares. Elle fut la résidence de tous les gouverneurs de la cité.» In : Poujol, 2001, p. 38.

18. PNB/habitant en 2004 au Tadjikistan : 1202 US\$ (1869 en Ouzbékistan, 7440 au Kazakhstan).

19. Discours du président d'Uzbektourism, M. Khakimov, le 3 octobre 2006, http://www.pressservices.uz/.

20. Cf. www.unescap.org

21. Enquête réalisée avec M. Z. K., le 8 septembre 2004 à Boukhara.

22. Observation sur le terrain, 2005.

23. Le procédé est déjà visible pendant la période soviétique. Cf. UNESCO, 1982, p. 13.

24. Cf. Nekrasova, Fillimonov, 1998, p. 95-124.

25. Entretien réalisé en russe avec M. Akhmedov, architecte de la province de Boukhara, le 18 octobre 2005, à Boukhara, avec l'aide de M. Z. K., architecte.

26. Aïvan : en ouzbek et tadjik, «élément architectural traditionnel dans les oasis d'Asie centrale, constitué d'un auvent soutenu par des colonnes en bois parfois sculpté, utilisé aussi bien pour les constructions publiques (tchaykhâna), sacrées (mosquées d'été, medressehs) que privées (maisons rurales et urbaines). » (Poujol C., 2001, p. 15).

\section{RÉSUMÉS}

Le « patrimoine urbain » est une expression utilisée en Europe occidentale depuis le début du XX siècle. Quelle signification prend-il en Asie centrale? Nous verrons que le patrimoine urbain y est un concept étatique qui répond à des impératifs économiques, politiques et non historiques. Il regroupe principalement des monuments utiles au pouvoir politique et se soucie peu de l'authenticité historique chère à l'Occident. 
The "urban heritage" is an expression used in Western Europe since the beginning of $\mathrm{XX}^{\text {th }}$ century. What meaning does it take in Central Asia? We shall see that the urban heritage is a state concept, which answers to economic, political and not historic imperatives. It includes mainly monuments useful for the political power and cares few about the historic authenticity advocated by the West.

\section{INDEX}

Mots-clés : Asie centrale, Etat, Ouzbékistan, patrimoine urbain, Xinjiang

Keywords : Central Asia, urban heritage, Uzbekistan, State, Xinjiang

\section{AUTEUR}

\section{GUILLEMETTE PINCENT}

Guillemette Pincent (pincentguillemette@gmail.com) est docteure et chercheuse associée au sein de l'ENEC. Elle a notamment publié ;

- «La reconstruction d'Eski Chahar à Tachkent et Boukhar : à la recherche d'un mythe?", Géographie et Cultures, $\mathrm{n}^{\circ}$ 65, numéro spécial sous la direction de M. le Professeur Augustin Berque (EHESS), « Une ville se refait-elle? », L'Harmattan, Paris, 2008, 12 p.

- «L'auto-construction dans les quartiers précoloniaux de Tachkent et de Boukhara : une dynamique urbaine à canaliser ? ", Les Cahiers de l'Asie centrale, Maisonneuve-Larose et IFEAC (Institut français d'études sur l'Asie centrale), Paris-Tachkent, 2007, nº 15-16, p. 291-306.

- « La réhabilitation fonctionnelle des villes précoloniales d'Ouzbékistan : un outil de domination politique? ", Cybergéo,

http://www.cybergeo.eu/index1750.html, juin 2006, 13 p. 